\begin{tabular}{|l|l|}
\hline $\begin{array}{l}\text { 2. To: (Receiving Organization) } \\
\text { Distribution }\end{array}$ & $\begin{array}{l}\text { 3. From: (Originating Organization) } \\
\text { TFRSO }\end{array}$ \\
\hline 5. Proj./Prog./Dept./Div.: & $\begin{array}{l}\text { 6. Design Authority/ Design } \\
\text { Agent/Cog. } \\
\text { Engr.: }\end{array}$ \\
W-314/RPP & J.S. Hammers \\
\hline
\end{tabular}

8. Originator Remarks:

This EDT Releases Specific Test and Evaluation Plans for Documents HNF-4578, HNF-4579, HNF-4580 and HNF-4581.

4. Related EOT No.:

7. Purchase Order No.:

N/A

9. Equip./Component No.

$\mathrm{N} / \mathrm{A}$

10. System/Bldg./Facility: 200E/241AN

11. Receiver Remarks: 11A. Design Baseline Document? [] Yes [X] No

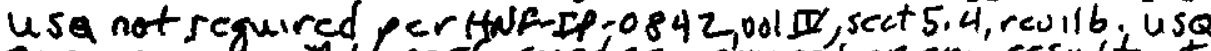
screenings will be performed as required on any resultant worll cuthorizing documants.

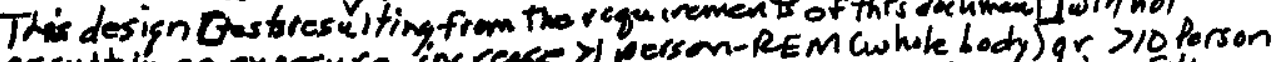
result in an exposure increace $>1$ person-REM W wole body) gr Rem (extremities) for the instellation, mainteumce andoperatioviof i) be hife of the modification, ALARA revieuss and regirements uilibe a ccomplished as partof the
12. Major Assm. Dwg. No.:

N/A

13. Permit/Permit Application No.:

$\mathrm{N} / \mathrm{A}$

14. Required Response Date:

N/A

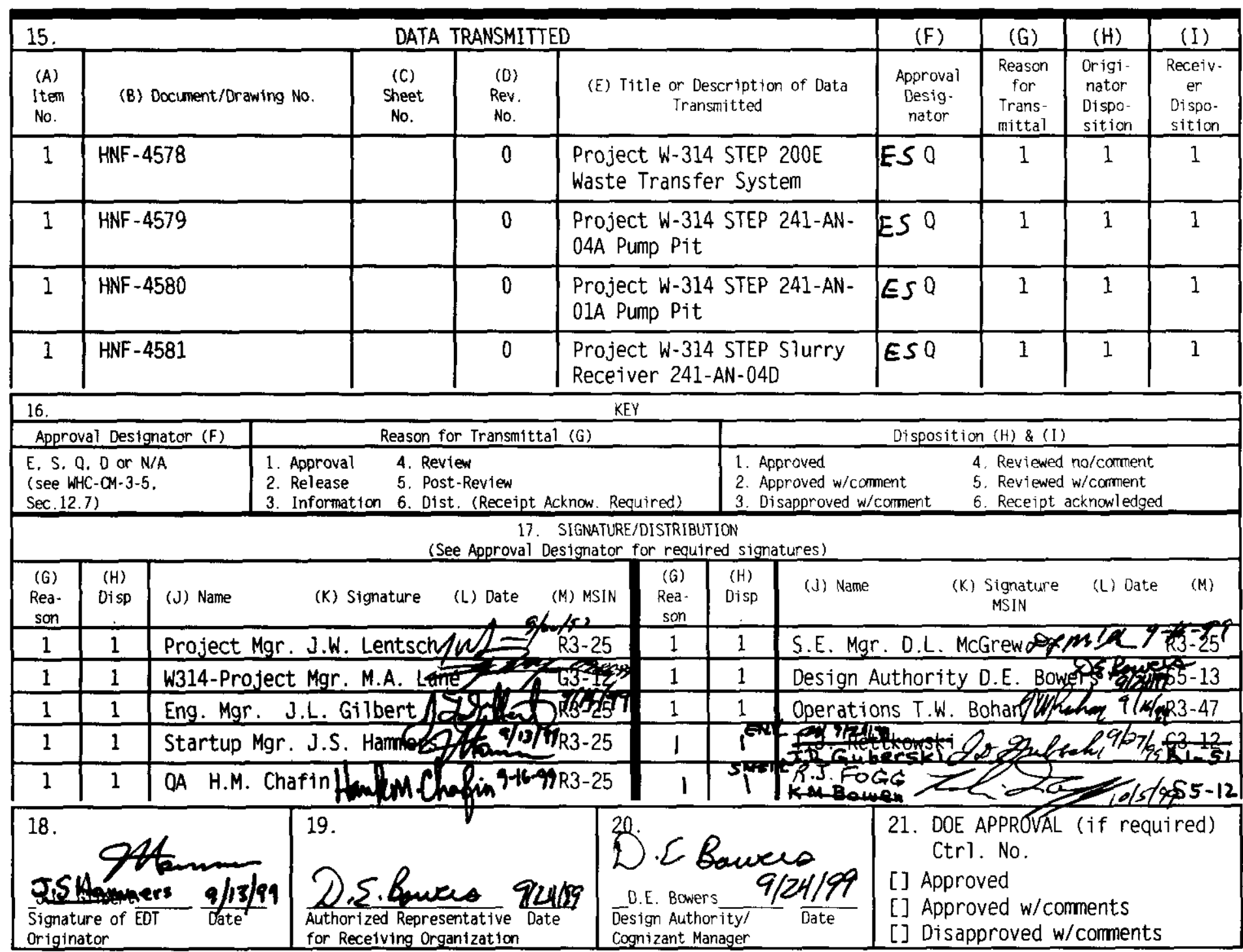




\section{Project W-314 Specific Test and Evaluation Plan for 200E Waste Transfer System}

Jack S. Hammers

Numatec Hanford Company, Richiand. WA 99352

U.S. Department of Energy Contract DE-AC06-96RL13200

EDT/ECN: 627805

Org Code: 8 C610

B\&R Code: EW3130010
UC: 2030

Charge Code: 106051

Total Pages:

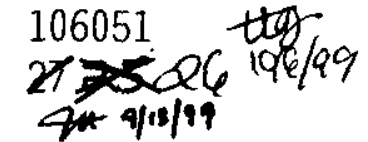

Key Words: Specific Test and Evaluation Plan. Project W-314, 200E Waste Transfer System

Abstract: This Specific Test and Evaluation Plan (STEP) defines the test \& evaluation activities performed on the 200E Waste Transfer System

TRADEMARK DISCLAIMER. Reference herein to any specific commercial product, process, or service by trade name. trademark, manufacturer, or otherwise, does not necessarily constitute or imply its endorsement. recomendation, or favoring by the United States Government or any agency thereof or its contractors or subcontractors.

Printed in the United States of America. To obtain copies of this document, contact: Document Control Services. P.0. Box 950. Mailstop H6-08. Richland WA 99352. Phone (509) 372-2420: Fax (509) 376-4989.
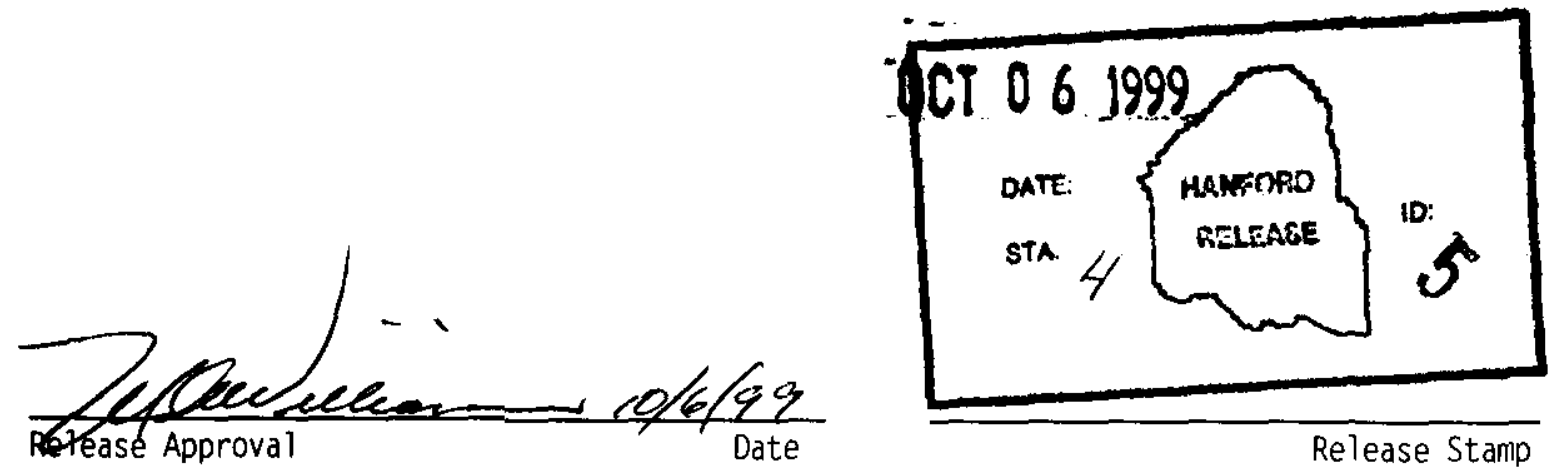

\section{Approved for Public Release}


HNF-4578, Rev. 0

\section{PROJECT W-314}

\section{SPECIFIC TEST AND EVALUATION PLAN \\ 200E WASTE TRANSEER SYSTEM}




\section{TABLE OF CONTENTS}

$1.0 \quad$ PURPOSE

2.0 SCOPE

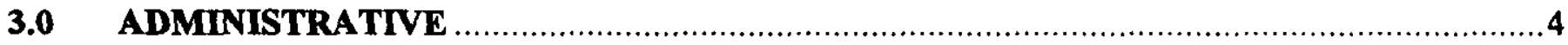

3.1 SAFETY INFORMATION/COMMUNICATIONS

4.0 GENERAL INFORMATION AND PREREQUISITES

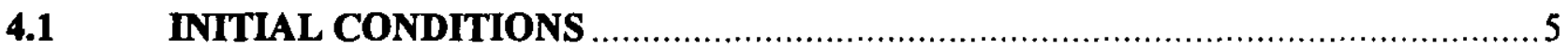

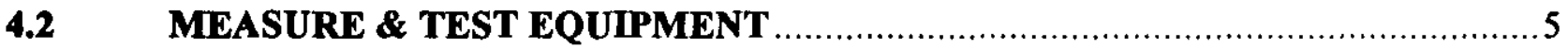

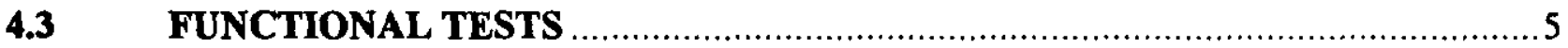

4.4 TEMPORARY MODIFICATIONS

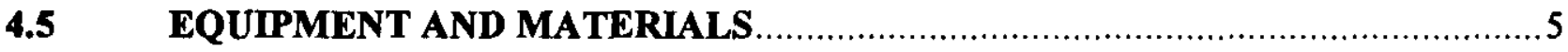

5.0 SCOPED TEST BOUNDARIES

5.1 OBJECTIVE

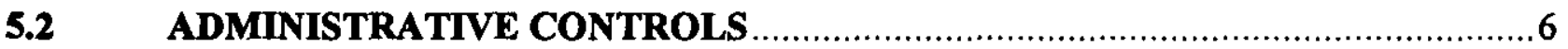

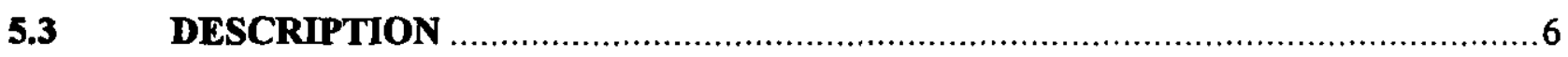

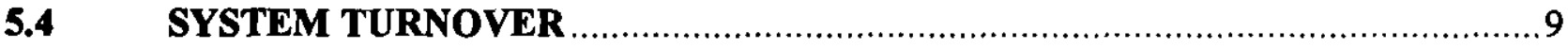

6.0 TEST AND ACCEPTANCE

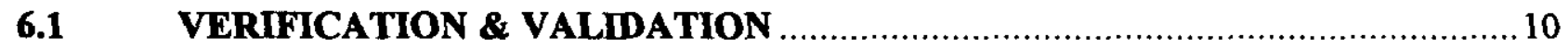

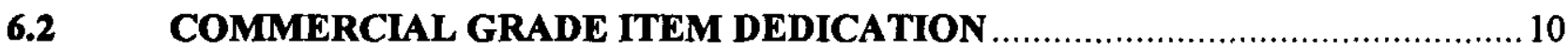

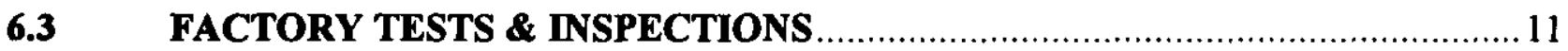

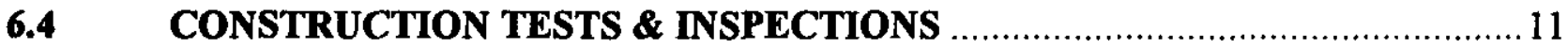

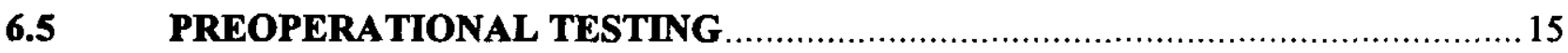

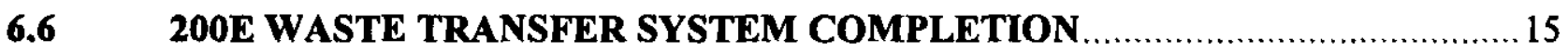

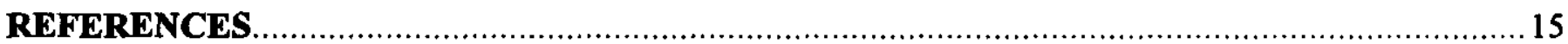

200E WASTE TRANFER SYSTEM COMPLETION CHECKLIST …....................................... 


\section{TABLE OF CONTENTS (CONT.)}

\begin{tabular}{|c|c|}
\hline \multicolumn{2}{|r|}{ LIST OF TABLES } \\
\hline TABLE 5-3 & 200E WASTE TRANSFER SYSTEM WORK AND TEST SCOPE DRAWINGS .6 \\
\hline TABLE 6-2 & COMMERCIAL GRADE ITEM DEDICATION ACTIVITIES ...................... 10 \\
\hline TABLE 6-3 & FACTORY TESTS \& INSPECTIONS \\
\hline TABLE 6-4 & CONSTRUCTION TESTS \& INSPECTIONS ........ \\
\hline TABLE 6-5 & PREOPERATIONAL TEST PROCEDURES \\
\hline FIGURE 1 & FLOW CHART 200E WTS \\
\hline FIGURE 2 & 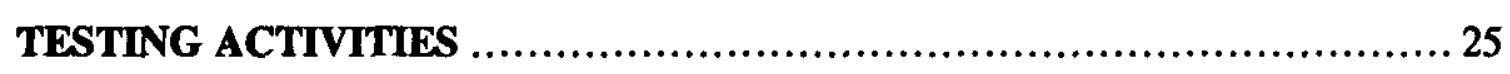 \\
\hline
\end{tabular}




\subsection{PURPOSE}

The purpose of this Specific Test and Evaluation Plan (STEP) is to provide a detailed written plan for the systematic testing of the newly constructed 200E Waste Transfer System in the W-314 Project. The STEP provides the outline for test and evaluation methods that verify the system's performance and compliance to the established Project design criteria. The STEP is a "lower tier" document based on the W-314 Test \& Evaluation Plan (TEP).

\subsection{SCOPE}

This STEP describes all testing and evaluation activities required for documenting compliance to the project design criteria as it relates to the construction of the 200E Waste Transfer System. This STEP identifies the specific testing and evaluation activities required by the Project Development Specifications (PDS), Procurement Specifications and Construction Specifications to define critical characteristics and attributes for Structures, Systems and components used to construct or modify the 200E Waste Transfer System in the W-314 Project Scope of Work. Testing or evaluation may include Validations and Verifications (e.g., Commercial Grade Item Dedication activities...etc), Factory Tests \& Inspections (FTIs), Construction Tests \& Inspections (CTIs), and Preoperational Test Procedures (POTPs). The STEP will be utilized in conjunction with the TEP for verification and validation.

\subsection{ADMINISTRATIVE}

\subsection{Safety Information/Communications}

Testing and inspection activities will be performed in various locations and facilities. The safety and communication procedures used to operate the area or facility (e.g., shop, tank farm, vendor facility...etc.) will be adhered to during the conduct of testing or inspection at these field locations. During the performance of 200 East Waste Transfer System field testing, the Operations Representative will be the direct line of communication with the Shift Office. During abnormal and casualty situations, the 200E Tank Farms Shift Manager will be the building emergency director/event commander. 


\subsection{GENERAL INFORMATION AND PREREQUISITES}

\subsection{Initial Conditions}

Prior to starting a test or demonstration the following conditions must be performed and verified:

- Prerequisite tests and construction activities, specific to the test, are completed.

- Test boundaries shall be defined and secure.

- Locks and tags are correctly placed to allow performance of the test.

- Test and support personnel are briefed on the scope and purpose of the test.

- Pre-job briefings are held, as required, before testing is conducted.

- Required test apparatus is functional and can be operated in accordance with safe practices during the test.

- Newly installed and existing equipment, within the boundary of the test, are operable and capable of operating in the testing environment.

\subsection{Measurement \& Test Equipment}

Measurement \& Test Equipment used will be specified per the test document and controlled per the providing group's Measurement \& Test Equipment program.

\subsection{Functional Tests}

Functional tests or demonstrations of Structures, Systems or Components, when required will be performed prior to conducting Preoperational Test Procedures. Functional tests and Demonstrations will be performed to approved procedures.

\subsection{Temporary Modifications}

Temporary modifications may be required in 200 East Tank Farms. Temporary modifications will be controlled per HNF-IP-0842, Vol. IV, section 4.5.

\subsection{Equipment and Materials}

Equipment installed by the contractor that fails during testing, or existing equipment that is damaged during the conduct of testing, will be the responsibility of the construction contractor. Existing facility Structures, Systems or Components found to be defective, will be the responsibility of River Protection Project (RPP) Operations. Rework or repair activities will be accomplished using an approved work package. 


\subsection{SCOPED TEST BOUNDARIES}

\subsection{Objective}

Startup scoped test boundaries are developed during the planning of the various tests listed in this STEP. These boundaries will define a testable system or component relationship to allow the conduct of testing in a safe configuration compatible with facility operating conditions.

\subsection{Administrative Controls}

The Lock and Tag system (HNF-IP-0842, Vol. II, Sections 4.9.1 and 4.10.1), as a minimum, will be utilized to establish the required administrative controls of energy sources.

\subsection{Description}

Table 5.3 lists the work and test scope drawings used for initial planning.

TABLE 5.3 - 200E WTS WORK AND TEST SCOPE DRAWINGS

\begin{tabular}{|c|c|c|c|}
\hline Bminio & 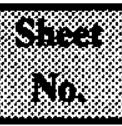 & rex & Tite \\
\hline H-2-68354 & 2 & 1 & Piping Plan Area \#3 \\
\hline H-14-102674 & 1 & 1 & Electrical Panelboard Schedule EDS-DP-709 \\
\hline H-14-103229 & 1 & 0 & Civil Project Site Plan \\
\hline H-14-103229 & 2 & 0 & Civil Project Temporary Fences \\
\hline H-14-103230 & 1 & 0 & Civil Buffalo \& 7th Intersection Plan \\
\hline$H-14-103231$ & 1 & 0 & Civil Typical Sections \& Details \\
\hline H-14-103232 & 1 & 0 & Civil Typical Section \& Details \\
\hline H-14-103233 & 1 & 0 & Structural Site Sections \& Details \\
\hline H-14-103233 & 2 & 0 & Civil 241-AZ-VP Site Preparation \\
\hline H-14-103234 & 1 & 0 & Civil WT -3150 \& WT -3160 Sta $0+00$ to Sta $5+50$ \\
\hline H-14-103235 & 1 & 0 & Civil WT -3150 \& WT- 3160 Sta $5+50$ to Sta $12+00$ \\
\hline H-14-103236 & 1 & 0 & Civil WT- 3150 \& WT- 3160 STA $12+00$ to Sta $17+00$ \\
\hline
\end{tabular}




\begin{tabular}{|c|c|c|c|}
\hline Prowing & sinet: & rer. & Tite \\
\hline H-14-103237 & 1 & 0 & Civil WT-3150 \& WT-3160 Sta $17+00$ to Sta END \\
\hline H-14-103238 & 1 & $\mathbf{0}$ & Civil SN-636 \& SN-630 Sta $0+00$ to Sta $6+75$ \\
\hline H-14-103239 & 1 & 0 & Civil SN-636, SN-637 \& SN-634 Sta $6+75$ to Sta $14+00$ \\
\hline $\mathrm{H}-14-103240$ & 1 & 0 & Civil WN-636, SN-637 \& SN-634 Sta $14+00$ to Sta $20+00$ \\
\hline H-14-103241 & 1 & 0 & Civil SN-636, SN-637 \& SN-634 Sta $20+00$ to Sta END \\
\hline H-14-103242 & 1 & 0 & Civil SN-634 Sta Equation to Sta END \\
\hline $\mathrm{H}-14-103243$ & 1 & C & Civil Project Temporary Fence \& Dwg. List \\
\hline H-14-103244 & 1 & A & Strl 241-AZ-VP Floor Plan \& General Notes \\
\hline $\mathrm{H}-14-103244$ & 2 & A & Strl 241-AZ-VP Sections \\
\hline $\mathrm{H}-14-103244$ & 3 & A & Strl 241-AZ-VP Pit Details \\
\hline H-14-103244 & 4 & A & Strl 241-AZ-VP Pit Details \\
\hline $\mathrm{H}-14-103245$ & 1 & A & Strl 241-AZ-VP Cover Blocks \\
\hline H-14-103245 & 2 & A & Str1 241-AZ-VP Cover Block Details \\
\hline H-14-103245 & 3 & A & Strl 241-AZ-VP Cover Block Details \\
\hline$H-14-103246$ & 1 & 0 & Civil 241-AZ-VP Site Preparations \\
\hline H-14-103246 & 2 & 0 & Strl Valve Pit Cover Blocks \\
\hline $\mathrm{H}-14-103247$ & 1 & 0 & Strl Leak Detector Station Missile Shield \\
\hline $\mathrm{H}-14-103250$ & 1 & 0 & Piping Jumper Arrangement Valve Pit 241-AZ-VP \\
\hline $\mathrm{H}-14-103250$ & 2 & 0 & Piping Jumper Arrangement Section A-A \\
\hline $\mathrm{H}-14-103250$ & 3 & 0 & Piping Jumper Arrangement Section B-B \\
\hline H-14-103251 & 1 & 0 & Piping Cross Site Transfer lines 244-A Tie-in details \\
\hline $\mathrm{H}-14-103253$ & 1 & 0 & Jumper Assembly Valve Pit AZ-VP-AZ-VP-A-H-J \\
\hline $\mathrm{H}-14-103253$ & 2 & 0 & Jumper Assembly Valve Pit AZ-VP-AZ-VP-A-H-J \\
\hline H-14-103254 & 1 & 0 & Jumper Assembly Valve Pit AZ-VP-AZ-VP-A-H-J \\
\hline $\mathrm{H}-14-103254$ & 2 & 0 & Jumper Assembly Vale Pit AZ-VP-AZ-VP-A-H-J \\
\hline H-14-103255 & 1 & 0 & Jumper Assembly Valve Pit AZ-VP-AZ-VP-E-F-(L) \\
\hline
\end{tabular}




\begin{tabular}{|c|c|c|c|}
\hline B & isicer: & $\mathrm{R}=2$ & Titie \\
\hline H-14-103255 & 2 & 0 & Jumper Assembly Valve Pit AZ-VP-AZ-VP-E-F-(L) \\
\hline H-14-103256 & 1 & 0 & Jumper Assembly Valve Pit AZ-VP-AZ-VP-C-K \\
\hline $\mathrm{H}-14-103257$ & 1 & 0 & Jumper Assembly Valve Pit AZ-VP-AZ-VP-B-Drain \\
\hline $\mathrm{H}-14-103258$ & 1 & 0 & Jumper Assembly Valve Pit AZ-VP-AZ-VP-D-Drain \\
\hline H-14-103259 & 1 & 0 & Jumper Assembly Valve Pit AZ-VP-AZ-VP-G-Drain \\
\hline H-14-103260 & 1 & 0 & Pit Cover Painting Diagrams Valve Pit 241-AZ-VP \\
\hline $\mathrm{H}-14-103260$ & 2 & 0 & Pit Cover Painting Diagrams Valve Pit 24l-AZ-VP \\
\hline $\mathrm{H}-14-103261$ & 1 & 0 & Funnel Assembly 2 and 3-Way Valves \\
\hline $\mathrm{H}-14-103261$ & 2 & 0 & Funnel Assembly Details \\
\hline H-14-103261 & 3 & 1 & Funnel Assembly Details \\
\hline $\mathrm{H}-14-103262$ & 1 & 0 & Valve handle Assembly 2 and 3-Way Valves \\
\hline H-14-103263 & 1 & 0 & Piping Valve Pit 241-AZ-VP Embedded Pipe Details \\
\hline H-14-103263 & 2 & 0 & Piping Valve Pit 241-AZ-VP Embedded Pipe Details \\
\hline $\mathrm{H}-14-103263$ & 3 & 0 & Piping Valve Pit 241-AZ-VP Embedded Pipe Details \\
\hline $\mathrm{H}-14-103263$ & 4 & 0 & Piping Valve Pit 241-AZ-VP Embedded Pipe Details \\
\hline H-14-103264 & 1 & 0 & Valve Actuator Arrangement Valve Pit 241-AZ-VP \\
\hline $\mathrm{H}-14-103265$ & 1 & 0 & Valve Actuator Details \\
\hline $\mathrm{H}-14-103265$ & 2 & 0 & Valve Actuator Details \\
\hline H-14-103266 & 1 & 0 & Piping Floor Drain Seal Assembly Valve Pit 241-AZ-VP \\
\hline H-14-103266 & 2 & 0 & Piping Floor Drain Seal Assembly Valve Pit 241-AZ-VP \\
\hline $\mathrm{H}-14-103274$ & 1 & $\mathrm{C}$ & INSTM Valve Pit Plan 241-AZ-VP \\
\hline $\mathrm{H}-14-103275$ & 1 & B & INSTM 241-AZ-VP Valve Pit Elevations \& Details \\
\hline $\mathrm{H}-14-103275$ & 2 & A & INSTM 241-AZ-VP Pump Pit Details \\
\hline $\mathrm{H}-14-103276$ & 1 & $\mathrm{C}$ & INSTM Valve Position Switches Loop Diagram \\
\hline $\mathrm{H}-14-103276$ & 2 & $\mathrm{C}$ & INSTM Valve Position Switches Loop Diagram \\
\hline H-14-103277 & 1 & C & INSTM Pump Pit Leak Detection LDE-221 Loop Diagram \\
\hline
\end{tabular}




\begin{tabular}{|c|c|c|c|}
\hline 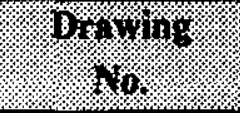 & \% & \% & 1410: \\
\hline H-14-103278 & 1 & $\mathbf{C}$ & INSTM ENCSD Low PT Leak DET LDE-222 Loop Diagram \\
\hline H-14-103279 & 1 & $\mathbf{C}$ & INSTM ENCSD Low PT Leak DET LDE-223 Loop Diagram \\
\hline H-14-103280 & 1 & B & INSTM ENCSD Low PT Leak DET LDE-224 Loop Diagram \\
\hline $\mathrm{H}-14-103281$ & 1 & B & INSTM Interconnection Diagram Field Terminal Box \\
\hline H-14-103281 & 2 & $\mathrm{C}$ & INSTM Interconnection Diagram Field Terminal Box \\
\hline$H-14-103281$ & 3 & $\mathrm{C}$ & INSTM Interconnection Diagram Field Terminal Box \\
\hline $\mathrm{H}-14-103282$ & 1 & $\mathrm{C}$ & INSTM Instrument List 241-AZ-VP Valve Pit \\
\hline $\mathrm{H}-14-103282$ & 2 & $\mathrm{C}$ & INSTM Instrument List 241-AZ-VP Valve Pit \\
\hline H-14-103282 & 3 & B & INSTM Instrument List 241-AZ-VP Valve Pit \\
\hline $\mathrm{H}-14-103283$ & 1 & $\mathbf{B}$ & INSTM MPS Terminal Box/Relay Encl. Plan \\
\hline $\mathrm{H}-\mathrm{I}$ - $\mathrm{I} 03284$ & 1 & B & NSTM MPS Box Plans \\
\hline H-14-103284 & 2 & B & INSTM MPS Box Elevations \\
\hline H-14-103285 & 1 & A & INSTM MPS field Terminal Box \\
\hline $\mathrm{H}-14-103286$ & 1 & $\mathrm{C}$ & INSTM MPS Relay Enclosure \\
\hline $\mathrm{H}-14-103287$ & 1 & $\mathrm{C}$ & INSTM Interconnection Diagram MPS Boxes \\
\hline $\mathrm{H}-14-103306$ & 1 & A & Electrical Power \& Control Plan 200-E WTS \\
\hline $\mathrm{H}-14-103306$ & 2 & A & Electrical Power \& Control Plan 200-E WTS \\
\hline $\mathrm{H}-14-103307$ & 1 & A & Electrical Power \& Control Plan AZ Valve Pit Area \\
\hline $\mathrm{H}-14-103308$ & 1 & A & Electrical Installation Details Valve Pit 241-AZ-VP \\
\hline $\mathrm{H}-14-103308$ & 2 & A & Electrical Installation Details Valve Pit 241-AZ-VP \\
\hline $\mathrm{H}-14-103310$ & 1 & A & Electrical Wire Run List 200E Waste Transfer System \\
\hline $\mathrm{H}-14-103311$ & 1 & A & Electrical Plans Profiles \& DET Power \& Signal Lines Mod. \\
\hline $\mathrm{H}-14-103312$ & 1 & B & Electrical Lighting Plan 241-AZ Tank Farm \\
\hline H-14-103313 & 1 & B & Electrical Power Plan 241-AZ Tank Farm \\
\hline
\end{tabular}




\subsection{System Turnover}

Jurisdiction Transfer, to RPP Operations, of Startup scoped Structures, Systems and Components will occur once testing has been completed and test results are reviewed and accepted. The SSC(s) will be transferred to RPP Operations as described in the W-314 TEP (HNF-SD-W314-TEP-001).

\subsection{TEST AND ACCEPTANCE}

The Project 200 East Waste Transfer System Construction test and evaluations are performed by various methods including engineering analysis, vendor data review, Factory Test $\&$ Inspections or Construction Tests \& Inspections defined in Procurement/Construction Specifications, engineering drawings, functional demonstrations, and Preoperational Tests. These verifications and tests will be documented in the W-314 Project 200E Waste Transfer System Requirements Verification Report (RVR), and in test evaluation reports.

\subsection{Validation and Verification}

Design requirements listed in the Project Development Specifications are reviewed by analysis and examination for project compliance. Commercial grade equipment utilized in a Safety Class application is qualified per appropriate procedures and programs. Equipment used in a non-safety class application is verified using vendor data to ensure the design requirements of the application are met.

\subsection{Commercial Grade Item Dedications}

Table 6-2 outlines testing that satisfies the Commercial Grade Item Dedication requirements. Development and execution responsibilities are in accordance with HNF-SD-W314-TEP-001.

TABLE 6-2

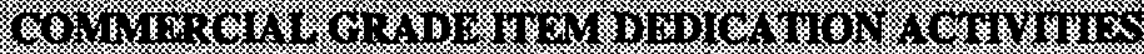

\begin{tabular}{|c|c|c|c|c|}
\hline TEST NUMBER & $\begin{array}{l}\text { TEST } \\
\text { NAME }\end{array}$ & $\begin{array}{l}\text { REFERENCE } \\
\text { DOCUMENT(S) }\end{array}$ & $\begin{array}{l}\text { ACCEPTANCE } \\
\text { DOCUMENT(S) }\end{array}$ & $\begin{array}{l}\text { ACCEPTANCE } \\
\text { NUMBER(S) }\end{array}$ \\
\hline 1.4.K._.CG.01 & $\begin{array}{l}\text { Reber Tensile Test and } \\
\text { Physical Inepection }\end{array}$ & $\begin{array}{l}\text { W-314-C6, Section 01400, 1.7, 03300, } \\
\text { 2.3.2 }\end{array}$ & & \\
\hline 1.4.K._CG.02 & $\begin{array}{l}\text { Stoel Plate, bar and shape } \\
\text { Tonsile toot and physical } \\
\text { Inspection }\end{array}$ & $\begin{array}{l}\text { W-314-C8, section } 01400,1.7,05500 \text {, } \\
\text { 1.3.2, 1.3.5, \& 2.3.2.1 }\end{array}$ & & \\
\hline 1.4.K._CG.03 & $\begin{array}{l}\text { Commercial Grado } \\
\text { Dedication for } \\
\text { instrumentation }\end{array}$ & $\begin{array}{l}W-314-C 6, \text { section } 01400,1.7,13440 \text {, } \\
\text { 1.2.3.2 }\end{array}$ & & \\
\hline 1.4.K._CG.04 & $\begin{array}{l}\text { Commercial Grade } \\
\text { Dodication for Piping } \\
\text { Matertals, verfify pipe size and } \\
\text { material }\end{array}$ & $\begin{array}{l}\text { W-314-C6, soction 01400, 1.7, } 15493 \text {, } \\
\text { 1.2.2.2 and 2.4; W-314-P3; and PDS-001 } \\
\text { 3.2.2.6, 3.3.1.15 }\end{array}$ & & \\
\hline 1.4.K._CG.05 & $\begin{array}{l}\text { Commercial Grade } \\
\text { Dedication for Electrical. }\end{array}$ & $\begin{array}{l}\text { W-314-C6, section 01400, 1.7, } 16400 \text {, } \\
\text { 1.2.2.1 }\end{array}$ & & \\
\hline
\end{tabular}




\subsection{Factory Tests \& Inspections}

Table 6-3 outlines the Factory Test \& Inspections that will be performed for $200 \mathrm{E}$ Waste Transfer System and Components. Development and execution responsibilities are in accordance with HNF-SD-W314-TEP-001.

TABLE 6-3

\begin{tabular}{|c|c|c|c|c|}
\hline $\begin{array}{l}\text { TEST } \\
\text { NUMBER }\end{array}$ & $\begin{array}{l}\text { TEST } \\
\text { NANE }\end{array}$ & REFERENCE DOCUMENT & $\begin{array}{l}\text { ACCEPTANCE } \\
\text { DOCUMENT(S) }\end{array}$ & $\begin{array}{l}\text { ACCEPTANCE } \\
\text { NUMBERS(S) }\end{array}$ \\
\hline 1.4. K_.F.01 & Valvo Hydro and Loak tests & $\begin{array}{l}\text { W-314-P1, section } 3.8 \& \text { W-314-P5 section } \\
3.9\end{array}$ & & \\
\hline 1.4.K._F.02 & Pipe welding & W-314-P3, section 3.8 & & \\
\hline 1.4.K._.F.03 & $\begin{array}{l}\text { Leak Delector and miac reloy } \\
\text { panels }\end{array}$ & $\begin{array}{l}\text { W-314P6, section 3.4, 3.5, 3.6, 4.1, 4.2, 4.38 } \\
\text { Appendix A }\end{array}$ & & \\
\hline
\end{tabular}

\subsection{Construction Tests \& Inspections}

Table 6-4 lists the construction tests performed during fabrication and installation to verify functionality of structures, systems and components. Development and execution responsibilities are in accordance with HNF-SD-W314-TEP-001.

TABLE 6-4

\begin{tabular}{|c|c|c|c|c|}
\hline TEST NUMBER & TEST & REFERENCE DOCUMENT & $\begin{array}{l}\text { ACCEPTANCE } \\
\text { DOCUNENT(S) }\end{array}$ & $\begin{array}{l}\text { ACCEPTANCE } \\
\text { NUMBER(S) } \\
\end{array}$ \\
\hline 1.4.K._.C.01 & In-Place Density Test & W-314-C6, section 02220, 3.7.1.2 & & \\
\hline 1.4.K._.C.02 & $\begin{array}{l}\text { Backfill Density Teat \& Cont. Soil } \\
\text { Use }\end{array}$ & $\begin{array}{l}\text { W-314-C6, Section 02220, 3.2, 3.3, 3.4, 3.5, } \\
\text { 3.7.1,: PDS-001, 3.7.3.2.1, PDS-002, 3.7.3.2.1 }\end{array}$ & & \\
\hline 1.4.K._.C.03 & Concrete Slump Test & $\begin{array}{l}\text { W-314-C6, section 03300, 2.2.1.4, 2.3.2.1, } \\
\text { 3.4.1; PDS-001, 3.3.1.4 POS-002, 3.3.4.2 }\end{array}$ & & \\
\hline 1.4.K._.C.04 & Concrete Alr Content Test & $\begin{array}{l}\text { W-314-C6, section } 03300,2.2 .1 .4,2.3 .2 .1 \text {, } \\
\text { 3.4.1; } \\
\text { PDS-001, 3.3.1.4; POS-002, 3.3.4.2 }\end{array}$ & & \\
\hline 1.4.K._.C.05 & Concrete Test Cylinders & $\begin{array}{l}\text { W-314-C6, section } 03300,2.2 .1 .4,2.3 .2 .1 \text {, } \\
3.4 .1 ; \\
\text { PDS-001, 3.3.1.4; PDS-002, 3.3.4.2 }\end{array}$ & & \\
\hline 1.4.K._.C.06 & $\begin{array}{l}\text { Concrete Preplecement } \\
\text { Inspections }\end{array}$ & $\begin{array}{l}\text { W-314-C6, section } 03300,3.1,3.2 .2 .1,3.2 .2 .7 \\
\text { \& 3.4.3; } \\
\text { PDS-001, 3.3.1.4; POS-002, 3.3.4.2 }\end{array}$ & & \\
\hline
\end{tabular}




\begin{tabular}{|c|c|c|c|c|}
\hline TEST NUMBER & TEST & REFERENCE DOCLIMENT & $\begin{array}{l}\text { ACCEPTANCE } \\
\text { DOCUMENT(S) }\end{array}$ & $\begin{array}{l}\text { ACCEPTANCE } \\
\text { NUMBER(S) }\end{array}$ \\
\hline 1.4.K._.C.07 & Concrete Placerment Inspections & $\begin{array}{l}\text { W-314-C6, section } 03300 \text { 3.2 \& 3.4; } \\
\text { PDS-001, 3.3.1.4; POS-002, 3.3.4.2 }\end{array}$ & & \\
\hline 1.4.K._.C.08 & $\begin{array}{l}\text { Concrete Post-Placement } \\
\text { Inspections }\end{array}$ & W-314C6, section $033003.2,3.3 \& 3.4$ & & \\
\hline 1.4.K._.C.09 & Expansion Anchor Inspections & W-314-C8, section 050553.4 .1 & & \\
\hline 1.4.K._.C.10 & Structural Wold Examinations & W-314-C6, section 05500, 3.5 & & \\
\hline 1.4.K._.C.11 & $\begin{array}{l}\text { Cover Block Special Protective } \\
\text { Coating Surface Preperation } \\
\text { Inspection }\end{array}$ & $\begin{array}{l}\text { W-314-C6, section 09855, 3.2 \& 3.4, PDS- } \\
002,3.7 .1 .2 .1 ; \text { PDS-005, 3.2.2.6, 3.2.2.7. } \\
\text { 3.3.1.2.1 }\end{array}$ & & \\
\hline 1.4.K._.C.12 & $\begin{array}{l}\text { Cover Block Special Profective } \\
\text { Coating Application }\end{array}$ & $\begin{array}{l}\text { W-314-C8, section 09855, 3.2.2, 3.2.3, 3.2.4, } \\
3.3,3.4, \text { PDS-002, 3.7.1.2.1; PDS-005, 3.2.2.1, } \\
\text { 3.2.2.2, 3.2.2.8, 3.2.4, 3.3.1.2.2 }\end{array}$ & & \\
\hline 1.4.K._.C.13 & $\begin{array}{l}\text { Pit Special Protective Coating } \\
\text { Surfece Preparation Inspection }\end{array}$ & $\begin{array}{l}\text { W.314-C6, section 09855, 3.2.2, 3.2.3, 3.2.4, } \\
\text { 3.3 \& 3.4, PDS-002, 3.7.1.2.1; POS-005, } \\
\text { 3.2.2.6, 3.3.1.2.1 }\end{array}$ & & \\
\hline 1.4.K._.C.14 & $\begin{array}{l}\text { Pit Special Protective Coeting } \\
\text { Application }\end{array}$ & $\begin{array}{l}\text { W-314-C6, section } 09855,3.2 .2,3.2 .3,3.2 .4 \text {, } \\
3.3,3.4, \text { PDS-002, 3.7.1.2.1; PDS-005, 3.2.2.1, } \\
\text { 3.2.2.2, 3.2.2.8, 3.2.4, 3.3.1.2.2 }\end{array}$ & & \\
\hline 1.4.K._.C.15 & Cover Block Painting Diegrams & $\begin{array}{l}\text { W-314-Ce, soction 09655, PDS-002, 3.3.3.1, } \\
\text { 3.7.1.2.12 }\end{array}$ & & \\
\hline 1.4.K._C.16 & $\begin{array}{l}\text { Leak Detector Fabrication Wiring } \\
\text { Continuity }\end{array}$ & $\begin{array}{l}\text { W-314-C6, section 13440, 3.3.1.1 \& section } \\
16400,3.3 .1 .1\end{array}$ & & \\
\hline 1.4.K._.C.17 & $\begin{array}{l}\text { Leak Detector Cabinet Installation } \\
\text { Wiring Continulity }\end{array}$ & $\begin{array}{l}\text { W-314-C6, eoction 13440, 3.3.1.1 \& section } \\
\text { 16400, 3.3.1.1 }\end{array}$ & & \\
\hline 1.4.K._.C.18 & $\begin{array}{l}\text { Cover Block installation Wiring } \\
\text { Continulity }\end{array}$ & $\begin{array}{l}\text { W-314-C6, eection 13440, 3.3.1.1 \& section } \\
18400,3.3 .1 .1\end{array}$ & & \\
\hline 1.4.K._.C.19 & $\begin{array}{l}\text { Terminal Box Conduit \& Cable } \\
\text { Installation Wiring Continulty }\end{array}$ & $\begin{array}{l}\text { W-314-C6, section 13440, 3.3.1.1 \& section } \\
\text { 16400, 3.3.1.1 }\end{array}$ & & \\
\hline 1.4.K._.C.20 & $\begin{array}{l}\text { Valve Limit Switch Wiring } \\
\text { Continuity }\end{array}$ & $\begin{array}{l}\text { W-314-C6, section 13440, 3.3.1.1 \& section } \\
\text { 16400, 3.3.1.1 }\end{array}$ & & \\
\hline 1.4.K._.C.21 & $\begin{array}{l}\text { Leak Delector Installation Wiring } \\
\text { Continuity }\end{array}$ & $\begin{array}{l}\text { W-314-C6, section 13440, 3.3.1.1 \& section } \\
16400,3.3 .1 .1\end{array}$ & & \\
\hline 1.4.K._.C.22 & $\begin{array}{l}\text { Cover Block Wiring Shielding } \\
\text { Resistance to Ground }\end{array}$ & W.314-C6, section $13440,3.2 .1 .2$ & & \\
\hline 1.4.K._.C.23 & $\begin{array}{l}\text { Terminal Box Conduit \& Cable } \\
\text { Shielding Resistance to Ground }\end{array}$ & W-314-C6, section 13440, 3.3.1.2 & & \\
\hline 1.4.K._.C.24 & $\begin{array}{l}\text { Valve Limit Swilch Shielding } \\
\text { Resistance to Ground }\end{array}$ & W-314-C6, ecetion 13440, 3.3.1.2 & & \\
\hline 1.4.K._.C.25 & $\begin{array}{l}\text { Leak Detector Shielding } \\
\text { Resistance to Ground }\end{array}$ & W-314-C6, section $13440,3.3 .1 .2$ & & \\
\hline 1.4.K._.C.26 & $\begin{array}{l}\text { Leak Detector Fabrication Voltage } \\
\text { Verification }\end{array}$ & W-314-C6, soction $16400,3.3 .1 .2$ & & \\
\hline
\end{tabular}




\begin{tabular}{|c|c|c|c|c|}
\hline TEST NUMBER & TEST & REFERENCE DOCUMENT & $\begin{array}{l}\text { ACCEPTANCE } \\
\text { DOCUMENT(S) }\end{array}$ & $\begin{array}{l}\text { ACCEPTANCE } \\
\text { NUMBER(S) }\end{array}$ \\
\hline 1.4.K._.C.27 & $\begin{array}{l}\text { Leak Detector Installation Voltago } \\
\text { Verification }\end{array}$ & W-314-C6, section 16400, 3.3.1.2 & & . \\
\hline 1.4.K._.C.28 & $\begin{array}{l}\text { Holiday Testing of Piping } \\
\text { Protective Costings }\end{array}$ & W-314-C6 section 15493, 3.1.6.3 & & \\
\hline 1.4.K._C.29 & $\begin{array}{l}\text { Piping Fabrication Welding } \\
\text { Non-Destructive Examination }\end{array}$ & $\begin{array}{l}\text { W-314-C6, section 15493, 3.3, W-314-P3; } \\
\text { PDS-001, 3.2.2.4, 3.3.1.5, 3.3.4; PDS-002, } \\
\text { 3.3.4 }\end{array}$ & & \\
\hline 1.4.K._.C.30 & $\begin{array}{l}\text { Jumper Fabrication Wolding } \\
\text { Non-Destructive Examination }\end{array}$ & $\begin{array}{l}\text { W-314-C8; W-314.P1, P3 \& PS } \\
\text { PDS-001, 3.3.1.5, 3.3.4; PDS-002, 3.3.4 }\end{array}$ & & \\
\hline 1.4.K._.C.31 & $\begin{array}{l}\text { Piping Fabrication Cleanliness } \\
\text { Inspection }\end{array}$ & $\begin{array}{l}\text { W-314-C6, section } 15493,3.2 .2 .4,3.2 .2 .5 ; W \text { - } \\
\text { 314-P3; PDS-001, 3.3.4 }\end{array}$ & & \\
\hline 1.4.K._.C.32 & Piping Fabrication Pressure Tests & $\begin{array}{l}\text { W-314-C6, section 15493, 3.2.3; W-314-P3; } \\
\text { PDS-001, 3.3.1.2 }\end{array}$ & . & \\
\hline 1.4.K._.C.33 & $\begin{array}{l}\text { Piping Installation Cleanliness } \\
\text { Inspection }\end{array}$ & $\begin{array}{l}\text { W-314-C6, section 15493, 3.2.2.5; PDS-001, } \\
\text { 3.3.4 }\end{array}$ & & \\
\hline 1.4.K._.C.34 & Piping installation Pressure Tests & $\begin{array}{l}\text { W-314-C6, section 15493, 3.2.3; POS-001, } \\
\text { 3.3.1.2 }\end{array}$ & & \\
\hline 1.4.K._.C.35 & Jumper Pressure Tests & W-314-C8; PDS-001, 3.3.1.2 & & \\
\hline 1.4.K._.C.36 & Jumper Fabrication Inspections & $\begin{array}{l}\text { W-314-C8; W-314-P1, P3 \& P5 } \\
\text { PDS-001, 3.3.1.5, 3.3.4; } \\
\text { PDS-002, 3.3.4 }\end{array}$ & & \\
\hline 1.4.K._.C.37 & $\begin{array}{l}\text { Jumper In service Leek Teet \& Pit } \\
\text { Configuration Verification }\end{array}$ & $\begin{array}{l}\text { HNF-SD-B10-001, section 5.3.2.18, TSR-006, } \\
\text { AC 5.12.2.a }\end{array}$ & & \\
\hline 1.4.K._.C.38 & $\begin{array}{l}\text { Leak Dotector Cabinet Inetallation } \\
\text { Electrical \& Instrumentation } \\
\text { Configuration \& Code Compliance }\end{array}$ & $\begin{array}{l}\text { PDS-001, 3.3.1.1, 3.3.4.10, 3.3.1.11, 3.3.1.12, } \\
\text { 3.7.2.2.2; PDS-003, 3.3.1. }\end{array}$ & & \\
\hline 1.4.K._C.39 & $\begin{array}{l}\text { Terminal Box Conduit \& Cable } \\
\text { Instellation Electrical \& } \\
\text { Instrumentation Configuration \& } \\
\text { Code Compllance }\end{array}$ & $\begin{array}{l}\text { PDS-001, 3.3.1.1, 3.3.1.10, 3.3.1.11, 3.3.1.12; } \\
\text { PDS-003, 3.3.1. }\end{array}$ & & \\
\hline 1.4.K._.C.40 & $\begin{array}{l}\text { Leak Detector Installation Electrical } \\
\text { Instrymentation Configuration \& } \\
\text { Code Compliance }\end{array}$ & $\begin{array}{l}\text { PDS-001, 3.3.1.1, 3.3.1.10, 3.3.1.11, 3.3.1.12, } \\
\text { 3.7.2.2.2; PDS-003, 3.2.1.4.2, 3.2.1.4.5, } \\
\text { 3.2.2.2, 3.3.1. }\end{array}$ & & \\
\hline 1.4.K._.C.41 & $\begin{array}{l}\text { Valve Limit Switch installation } \\
\text { Electriceal \& Instrumentation } \\
\text { Configuration \& Code Compliance }\end{array}$ & $\begin{array}{l}\text { PDS-091, 3.3.1.1, 3.3.1.10, 3.3.1.11, 3.3.1.12, } \\
\text { 3.7.2.2.2; PDS-003, 3.3.1. }\end{array}$ & & \\
\hline 1.4.K._.C.42 & $\begin{array}{l}\text { Piping Installation Conflguration } \\
\text { Verfication }\end{array}$ & PDS-001, 3.2.2, 3.3.1.15 & & \\
\hline 1.4.K._.C.43 & Cathodic Protection & N/A & & \\
\hline 1.4.K._.C.44 & Jumper Fit Up \& Installation & RPP Operations Requirement & & \\
\hline 1.4.K._.C.45 & Cover Block Fit \& Installation & RPP Operations Requirement & & \\
\hline 1.4.K._.C.46 & $\begin{array}{l}\text { Load test Cover Block lifting beils \& } \\
\text { Lifting beil Magnetic Particle Teet }\end{array}$ & $\begin{array}{l}\text { W-314-C6, section } 03300,3.2 .5 \\
\text { PDS-002, 3.3.4.2 }\end{array}$ & & \\
\hline
\end{tabular}




\begin{tabular}{|c|c|c|c|c|}
\hline TEST NUMBER & TEST & REFERENCE DOCUMENT & $\begin{array}{l}\text { ACCEPTANCE } \\
\text { DOCUMENT(S) }\end{array}$ & $\begin{array}{l}\text { ACCEPTANCE } \\
\text { NUMBER(S) }\end{array}$ \\
\hline 1.4.K._.C.47 & Jumper support leg adjustment & $\begin{array}{l}\text { H-14-103253, SH 1, Note7 } \\
\text { H-14-103254, SH1, Note 7 } \\
\text { H-14-103255, SH1, Note 7 } \\
\text { H-14-103256, SH1, Note } 7 \\
\text { H-14-103257, SH1. Note } 7 \\
\text { H-14-103258, SH1, Noto } 7 \\
\text { H-14-103259, SH1, Noto } 7\end{array}$ & & \\
\hline 1.4.K._.C.48 & $\begin{array}{l}\text { Final Special Prolectwo Coeting } \\
\text { inspection of pit \& cower block }\end{array}$ & W-314 Project Management requirement & & \\
\hline 1.4.K._.C.49 & Concrete Embedment Installation & W-314-C6, section $03300,3.4 .3$ & & \\
\hline 1.4.K._.C.50 & Leak Detection Reloy Cobinets & W-314-C6, section $13440,3.1 .3$ & & \\
\hline 1.4.K._.C.51 & $\begin{array}{l}\text { Intrinsically Safo Wiring Color } \\
\text { Coded/Labelod }\end{array}$ & W-314-C6, 13440, Section 3.1.4 & & \\
\hline 1.4.K._.C.52 & Intrineically Salo Wiring & W-314-C6, eaction 13440, 3.1.4 & & \\
\hline $1.4 . K . . C .53$ & Valvos & $\begin{array}{l}\text { W-314-C6, section } 15493,3.2 .4 .1,3.2 .4 .2 \text {, } \\
3.2 .4 .3 ; \text { W-314-C8; W-314-P1 \& P5; POS-Co2, } \\
3.2 .2 .1,3.2 .2 .2,3.2 .2 .5, \quad 3.2 .2 .7,3.2 .2 .8\end{array}$ & & \\
\hline 1.4.K._.C.54 & Laboling & $\begin{array}{l}\text { W-314-C6, section 16400, 1.3.16, PDS-002, } \\
\text { 3.3.3.1; POS-003, 3.3.3.1, 3.3.3.4 }\end{array}$ & & \\
\hline 1.4.K._.C.55 & Enclosure Type & W-314-C6, section 16400, 2.2 .9 & & \\
\hline 1.4.K._.C.56 & $\begin{array}{l}\text { Painted Surface Preparation } \\
\text { Inspection }\end{array}$ & W-314-C6, section 09900, 3.2.2, \& 3.4 & & \\
\hline 1.4.K._.C.57 & Paint Application & W-314-C6, section 09900, $3.3 \& 3.4$ & & \\
\hline $1.4 . K . . C .58$ & $\begin{array}{l}\text { Stainless Stoel Pit Liner } \\
\text { Preinstallation }\end{array}$ & W-314-C6, eaction 05555, 1.3.1, & & \\
\hline 1.4.K._.C.59 & Stainloses Stool Pit Liner Installetion & $\begin{array}{l}\text { W-341-C6, section } 05555,3.1,3.2,3.3,3.48 \\
3.5\end{array}$ & & \\
\hline 1.4.K._.C.60 & Roed bed Preparation & W-314-C6, section $02235,3.1,3.2 \& 3.6$ & & \\
\hline 1.4.K._C.61 & Asphalt Application & W-314-C6, section 02512, 3.1, 3.2 & & \\
\hline 1.4.K._.C.62 & Buried Pipe & $\begin{array}{l}\text { POS-001, 3.3.1.5, 3.3.1.6, 3.3.1.7, 3.7.1.2.1, } \\
\text { 3.7.3.2.2 }\end{array}$ & & \\
\hline 1.4.K._.C.63 & Valvo Ph Construction & $\begin{array}{l}\text { PDS-002, 3.7.1.1.9, 3.7.1.2.1, 3.7.1.2.2, } \\
\text { 3.7.1.2.3, 3.7.1.2.12 }\end{array}$ & & \\
\hline 1.4.K._.C.64 & Valvo PH Cover Block inapection & POS-002, 3.7.1.2.5 - 3.7.1.2.12 & & \\
\hline 1.4.K._C.65 & Area Lighting Inspections & $\begin{array}{l}\text { PDS- }-02, \text { 3.7.3.1.3, 3.7.3.1.4 3.7.3.2.2, } \\
\text { 3.7.3.2.3 }\end{array}$ & & \\
\hline
\end{tabular}




\subsection{Preoperational Testing}

Table 6-5 lists the preoperational tests performed to demonstrate Structure, System and Components function in accordance with engineering requirements. Development and execution responsibilities are in accordance with HNF-SD-W314-TEP-001

TABLE 6-5

\begin{tabular}{|c|c|c|c|c|}
\hline \multicolumn{5}{|c|}{ 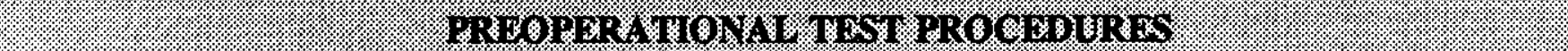 } \\
\hline TEST DOCUMENT & TEST DESCRIPTION & REFERENCE DOCUMENT & $\begin{array}{l}\text { ACCEPTANCE } \\
\text { DOCUMENT(S) }\end{array}$ & $\begin{array}{l}\text { ACCEPTANCE } \\
\text { NUMBER(S) }\end{array}$ \\
\hline 1.4.K._.T1 & Encasement Leak Detection & 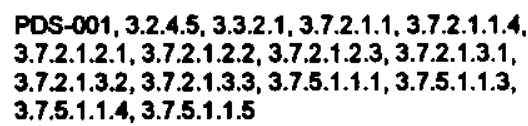 & & \\
\hline 1.4.k._.T2 & AZ Valve Plt Loak Delection & $\begin{array}{l}\text { PDS-003, 3.2.1.1.1, 3.2.1.1.2, 3.2.1.1.3, } \\
\text { 3.2.1.1.4, } 3.2 .1 .1 .5,3.2 .1 .1 .6,3.2 .1 .2 .1 \\
\text { 3.2.1.2.2, 3.2.1.2.3, 3.2.1.3.1, 3.2.1.3.2, } \\
\text { 3.2.1.3.3, 3.2.1.4.1, 3.2.1.4.3, 3.2.4.4, 3.3.2.1 }\end{array}$ & & \\
\hline 1.4.k._T3 & Valve Manifold and indication & $\begin{array}{l}\text { POS-002, 3.2.1.1.1, 3.2.2.1a, 3.2.2.1b, 3.2.2.2, } \\
\text { 3.3.2.1, 3.3.7.3, 3.7.2.2.1 }\end{array}$ & & \\
\hline
\end{tabular}

\subsection{E Waste Transfer System Completion}

200E Waste Transfer System testing will be considered successfully completed when the TEST COMPLETION CHECKLIST (attached to the end of this STEP) is completely signed off. This checklist is a "stand alone" document that will be listed on the 200E Waste Transfer System ABU) and included in the turnover of documents. This checklist will be completed by the W-314 Startup Manager or his designee.

\section{REFERENCES}

HNF-PRO-1819, PHMC Engineering Requirements, Fluor Daniel Hanford Company, Richland, Washington.

HNF-PRO-2000, Construction Program Execution Phase, Fluor Daniel Hanford Company, Richland, Washington.

W-314-P1, Procurement Specification Ball Valves AN Valve Pit Upgrades, Fluor Daniel Northwest, Inc., Richland, Washington.

W-314-P3, Procurement Specification Design and Fabrication for a Double-containment Piping System, Fluor Daniel Northwest, Inc., Richland, Washington.

W-314-P5 Procurement Specification 3-Inch Block Valves, Fluor Daniel Northwest, Inc., Richland, Washington. 
W-314-P6, Procurement Specification Leak Detector Panel Fabrication, Fluor Daniel Northwest, Inc., Richland, Washington.

HNF-SD-W314-PDS-001, Project Development Specification - Transfer Piping, Fluor Daniel Northwest, Inc., Richland, Washington.

HNF-SD-W314-PDS-002, Project Development Specification for Valve Pit Manifold, Fluor Daniel Northwest, Inc., Richland, Washington.

HNF-SD-W314-PDS-003, Project Development Specification for Pit Leak Detection, Fluor Daniel Northwest, Inc., Richland, Washington.

HNF-SD-W314-PDS-005, Project Development Specification for Special Protective Coating, Fluor Daniel Northwest, Inc., Richland, Washington.

HNF-SD-W314-TEP-001, Test and Evaluation Plan for W-314 Tank Farm Restoration and Safe Operations, Numatec Hanford Corporation, Richland, Washington.

HNF-IP-0842, RPP Administration, Fluor Daniel Hanford Company, Richland, Washington.

HNF-IP-1266, Tank Farm Operations Administrative Controls, Fluor Daniel Hanford Company, Richland, Washington.

W-314-C6-1, Acceptance Inspection Plan, Fluor Daniel Hanford Corporation, Richland, Washington.

W-314-C6, Construction Specification 200 East Waste Transfer System, Fluor Daniel Northwest, Inc., Richland, Washington.

W-314-C7, Construction Specification 200 East Waste Transfer System (W-058 Tie-In), Fluor Daniel Northwest, Inc., Richland, Washington.

W-314-C8, Construction Specification Jumper Fabrication, Fluor Daniel Northwest, Inc., Richland, Washington. 


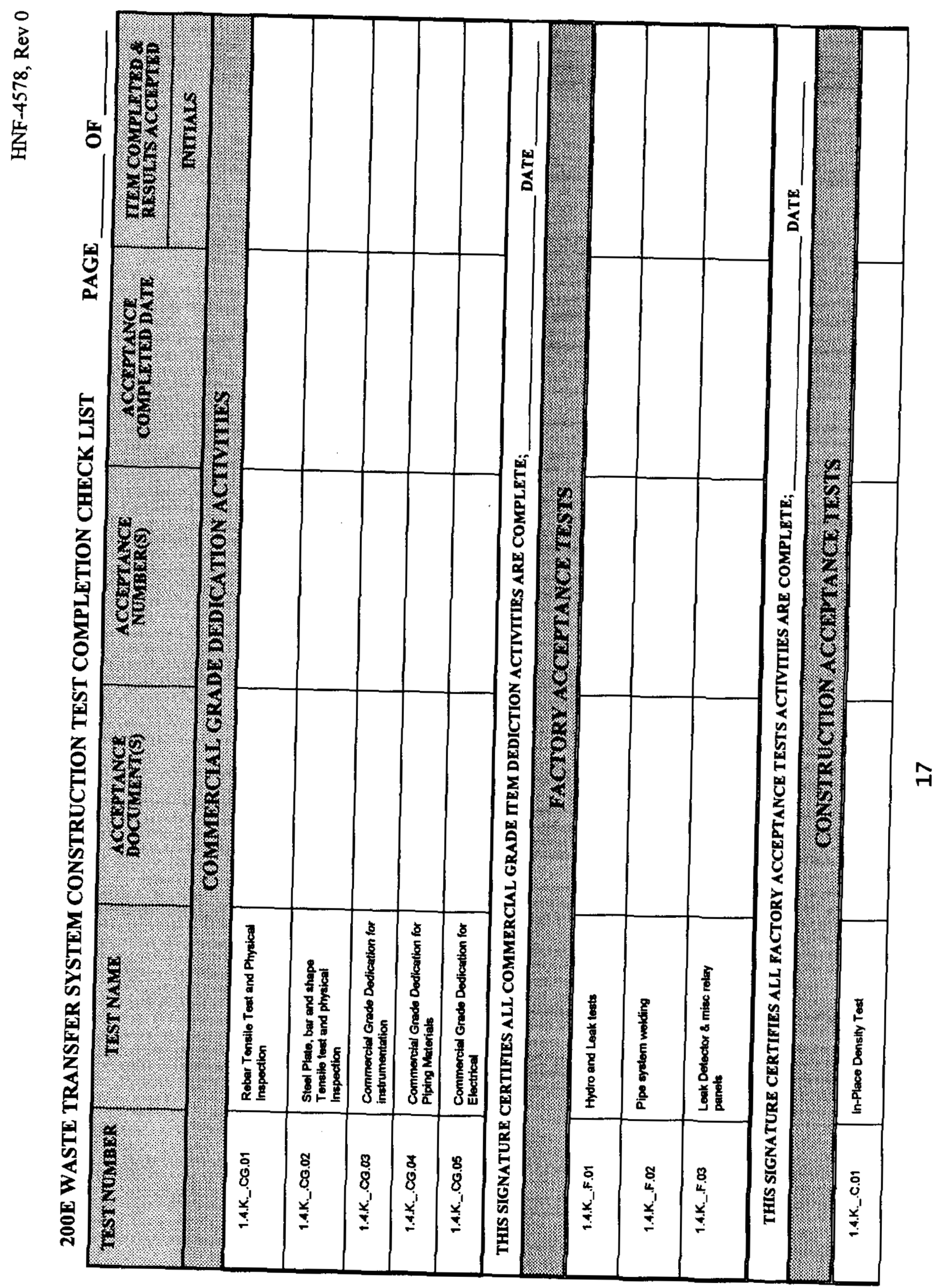




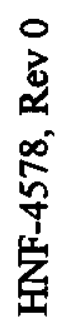

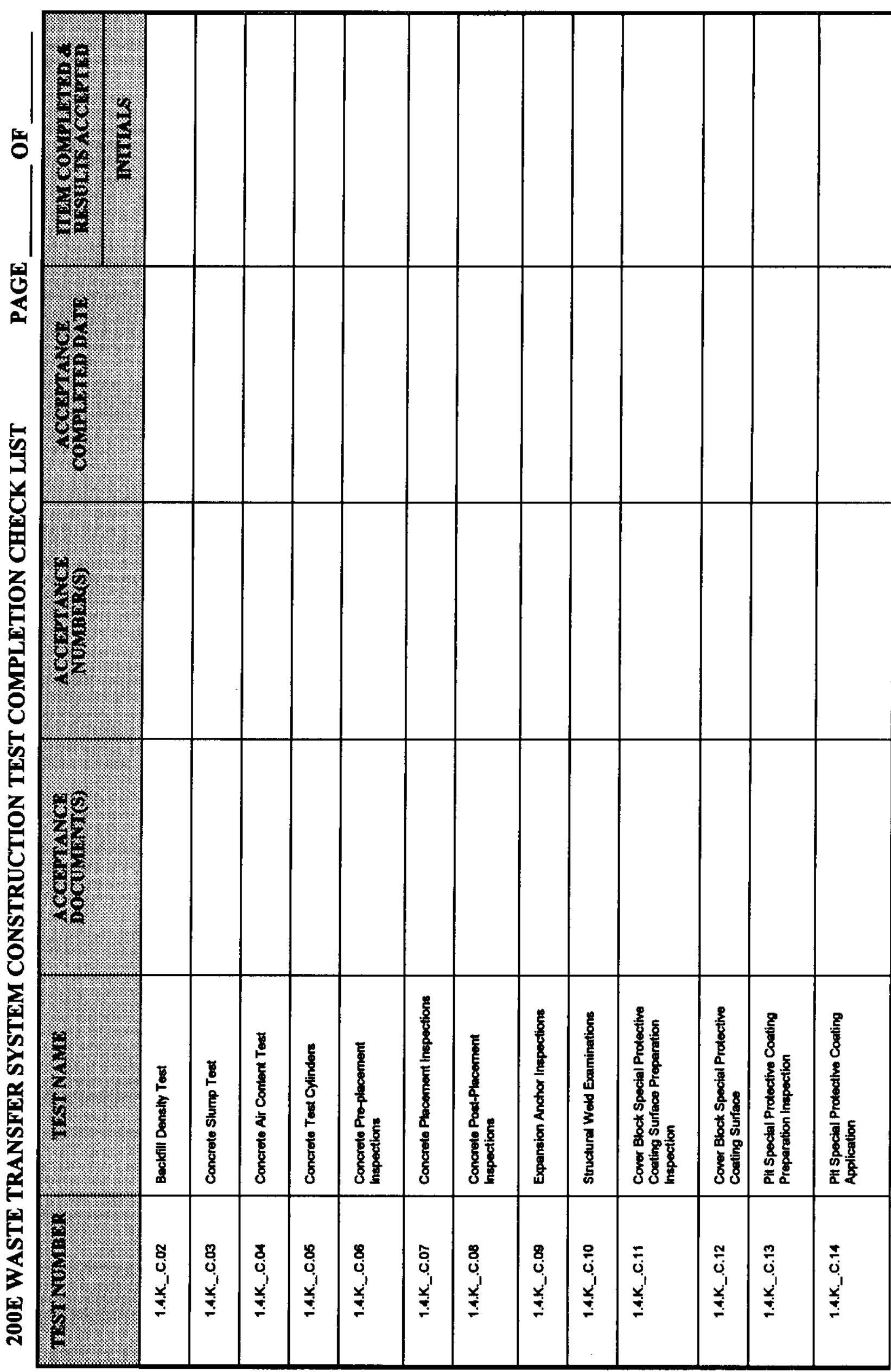




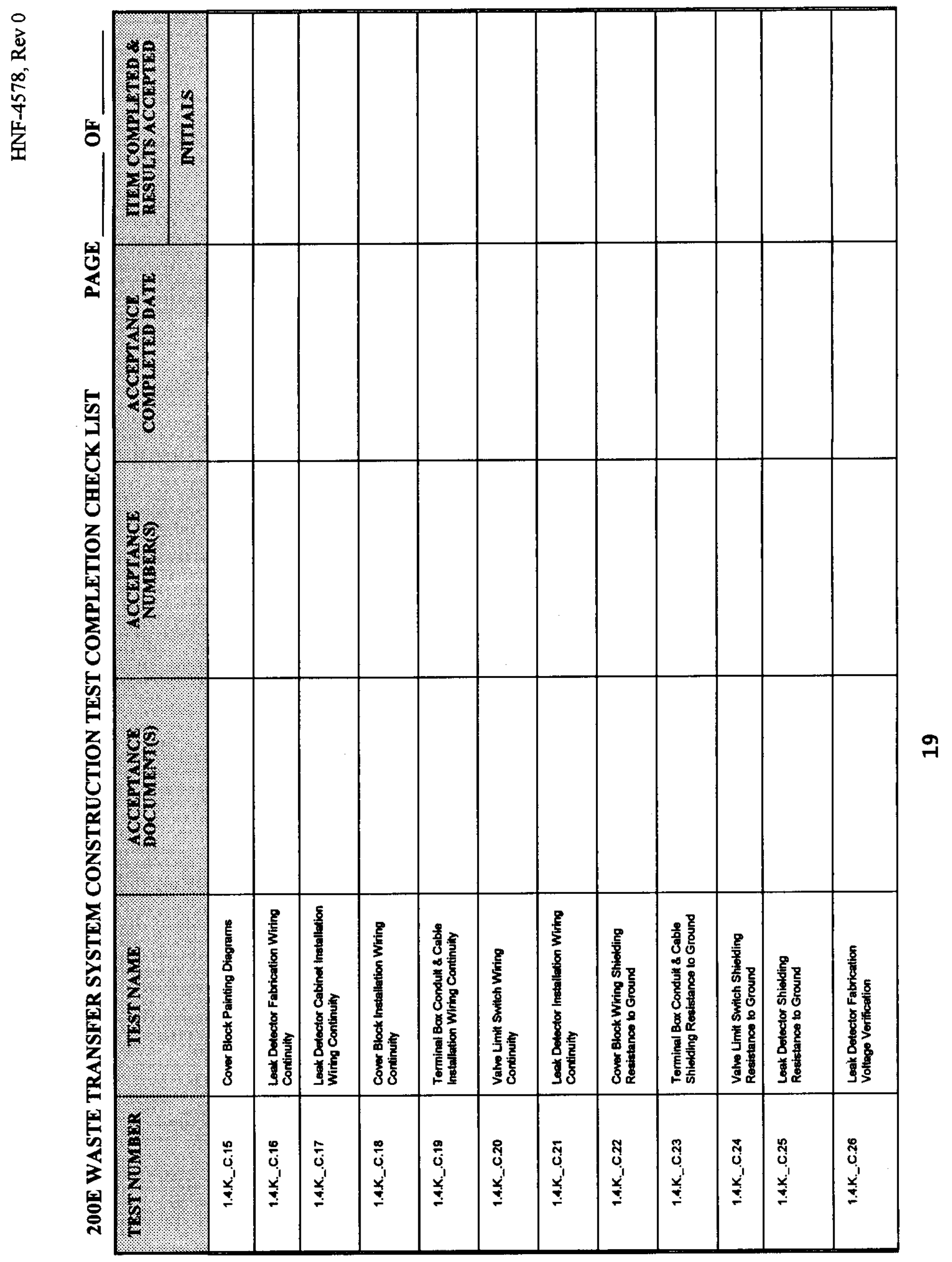




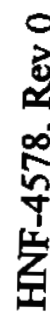

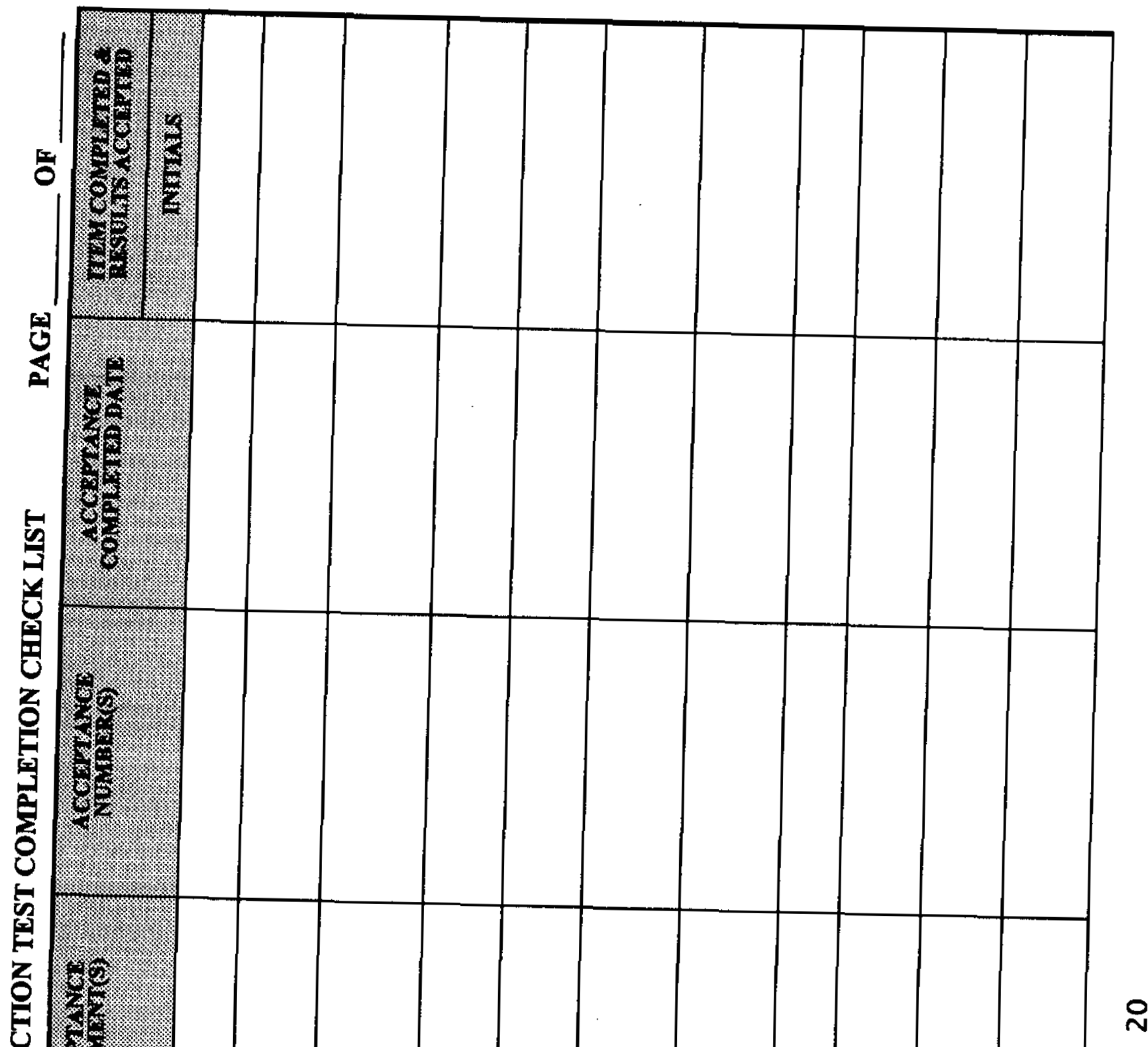




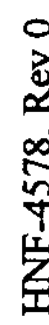

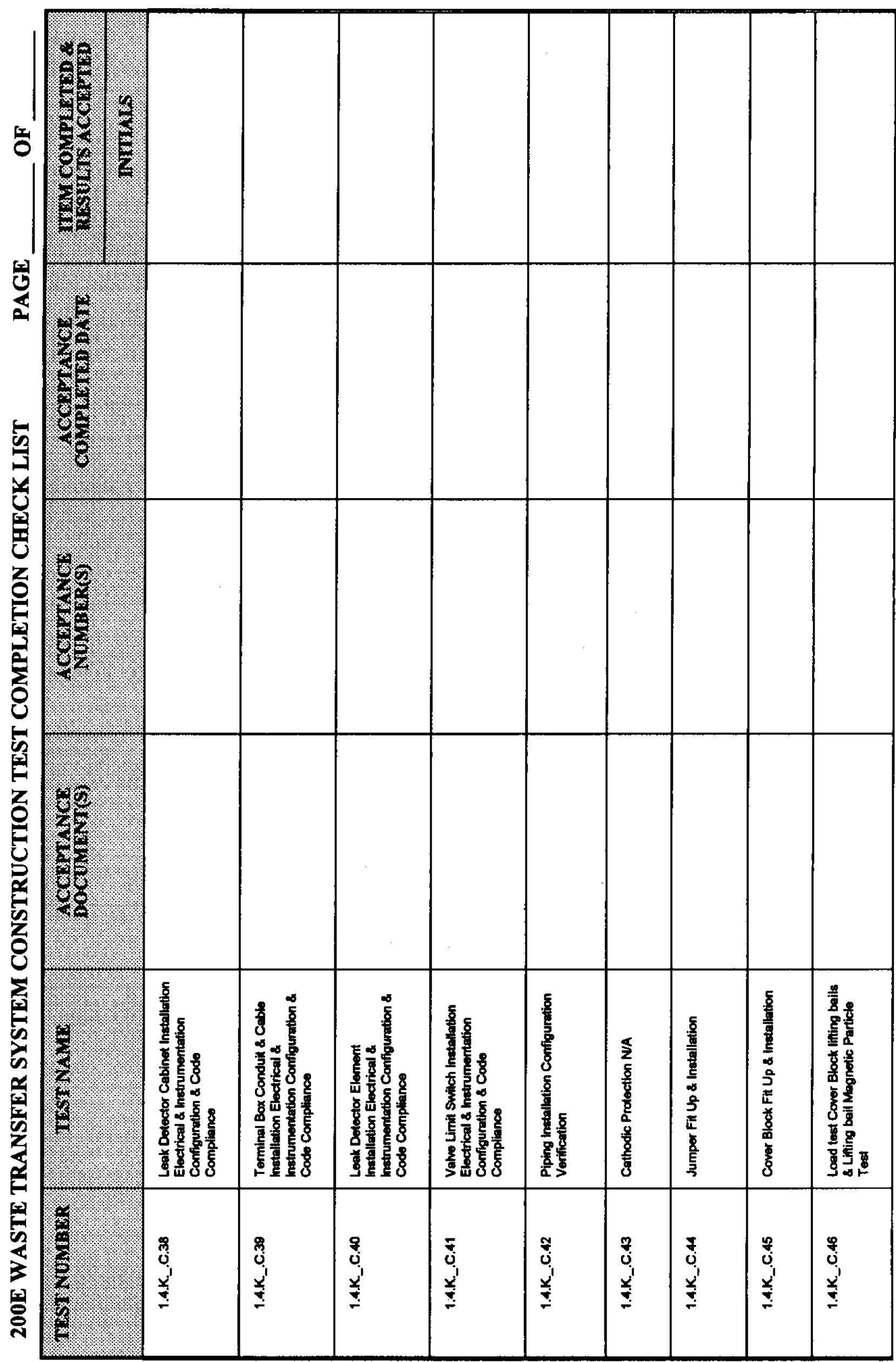

$N$ 


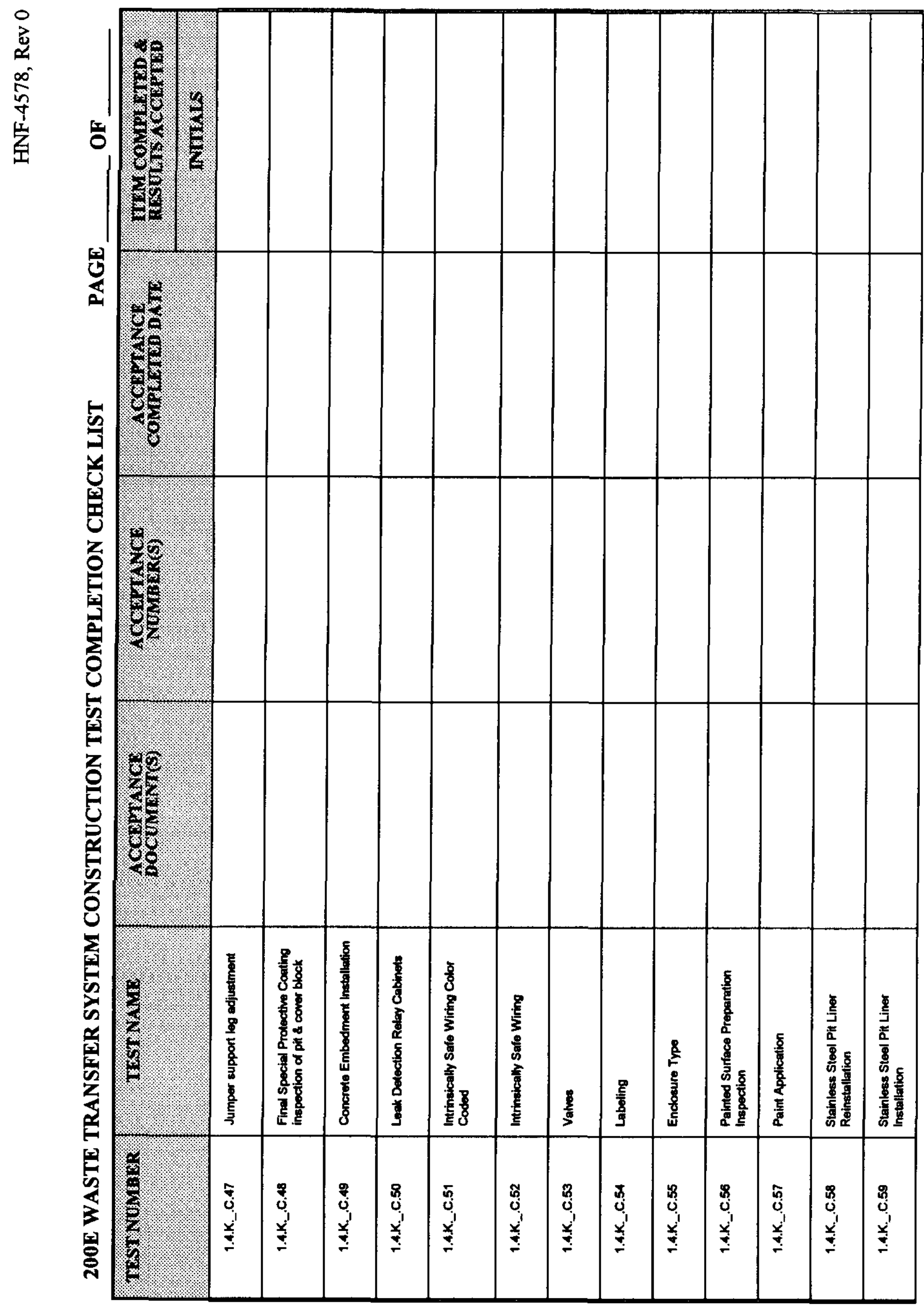

$N$ 


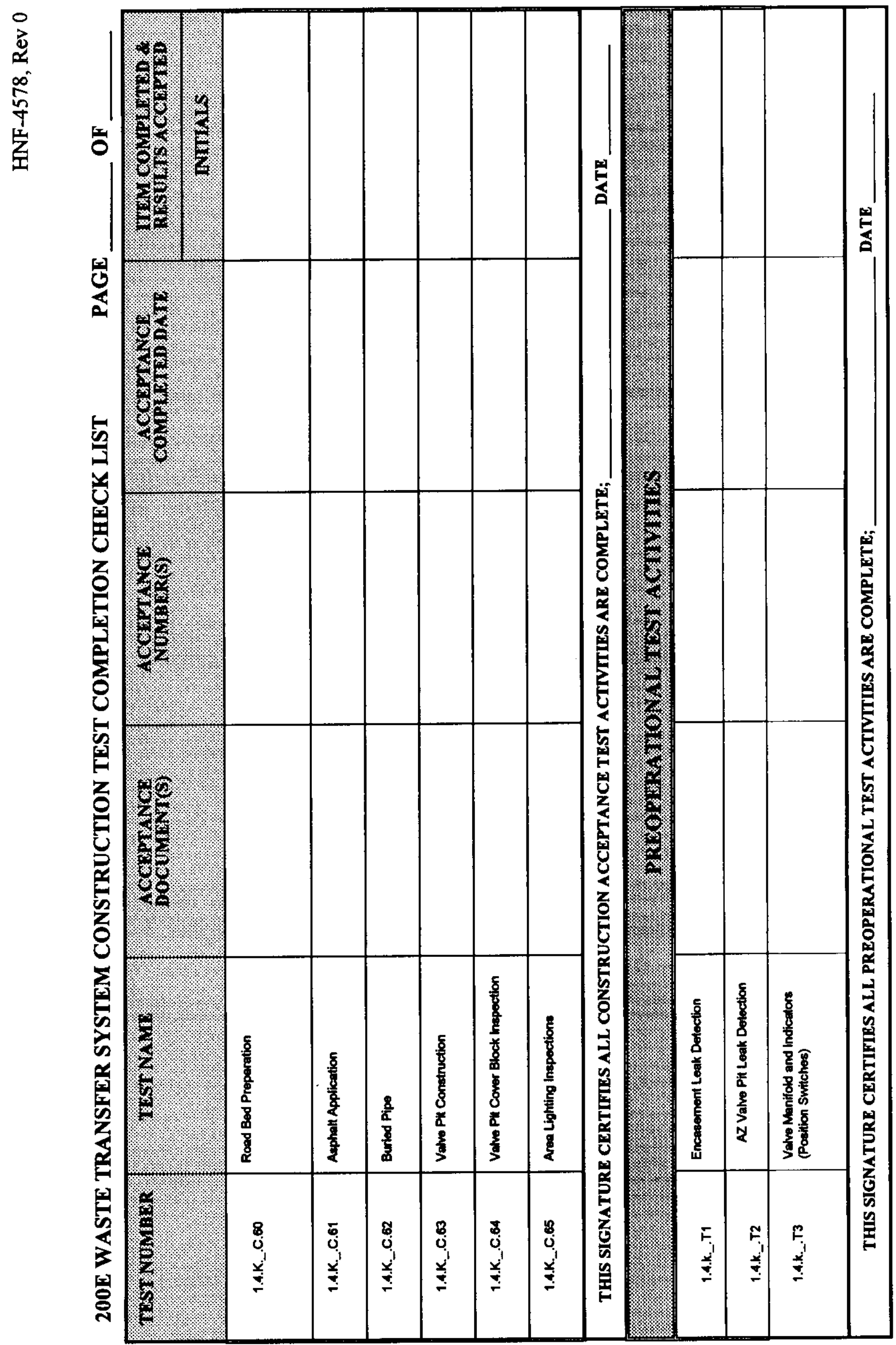

$\stackrel{m}{N}$ 


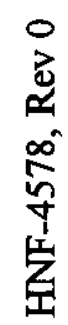

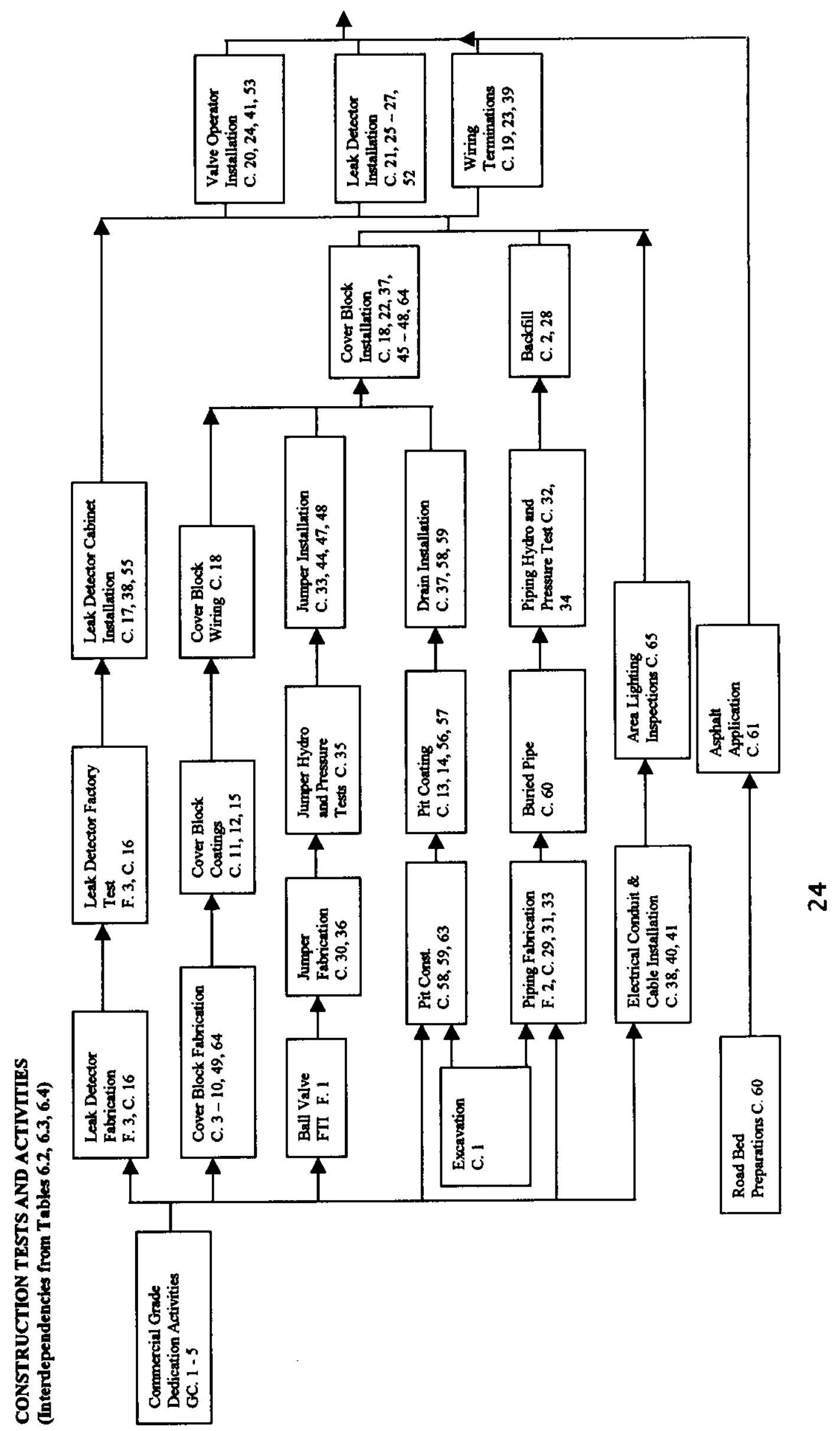




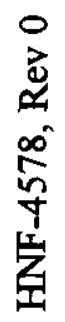

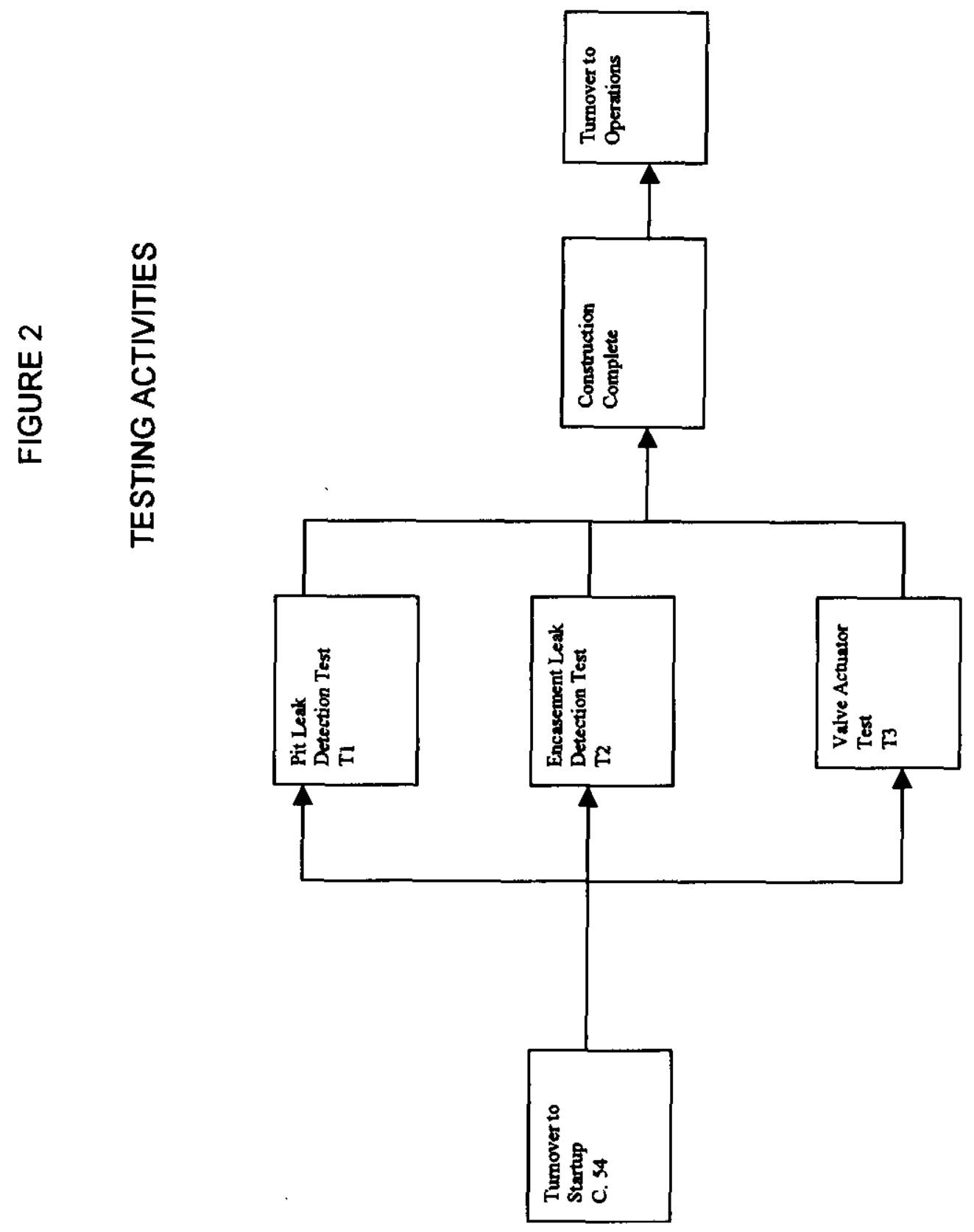

n 